\title{
Integration of Holomorphic Lie Algebroids
}

\author{
Camille Laurent-Gengoux \\ Département de mathématiques \\ Université de Poitiers \\ 86962 Futuroscope-Chasseneuil, France \\ laurent@math.univ-poitiers.fr \\ Mathieu Stiénon * \\ E.T.H. Zürich \\ Departement Mathematik \\ 8092 Zürich, Switzerland \\ stienon@math.ethz.ch \\ Ping $\mathrm{Xu}{ }^{\dagger}$ \\ Department of Mathematics \\ Penn State University \\ University Park, PA 16802, U.S.A. \\ ping@math.psu.edu
}

\begin{abstract}
We prove that a holomorphic Lie algebroid is integrable if, and only if, its underlying real Lie algebroid is integrable. Thus the integrability criteria of Crainic-Fernandes (Theorem 4.1 in [6]) do also apply in the holomorphic context without any modification. As a consequence we gave another proof of the theorem that a holomorphic Poisson manifold is integrable if and only if its real part or imaginary part is integrable as a real Poisson manifold [5, 20.
\end{abstract}

\section{Contents}

\section{Introduction}

2 Holomorphic Lie algebroids 4

2.1 Holomorphic Lie algebroids . . . . . . . . . . . . . . . . . . . 4

2.2 Tangent Lie algebroids . . . . . . . . . . . . . . . . . . . 5

2.3 Equivalent definition of holomorphic Lie algebroids . . . . . . . . . . . 7

2.4 Holomorphic Poisson manifolds . . . . . . . . . . . . . . . . . . . . . . . 9 9

\footnotetext{
${ }^{*}$ Research supported by the European Union through the FP6 Marie Curie R.T.N. ENIGMA (Contract number MRTN-CT-2004-5652).

${ }^{\dagger}$ Research partially supported by NSF grants DMS-0306665 and DMS-0605725 \& NSA grant H98230-06-10047
} 
3 Holomorphic Lie groupoids and holomorphic symplectic groupoids

3.1 Multiplicative $(1,1)$-tensors on Lie groupoids . . . . . . . . . . . . . 10

3.2 Nijenhuis torsion of multiplicative $(1,1)$-tensors $\ldots \ldots \ldots \ldots$

3.3 Infinitesimal multiplicative $(1,1)$-tensors $\ldots \ldots \ldots \ldots$. . . . . . . . . . 14

3.4 Multiplicative Nijenhuis tensors on Lie groupoids . . . . . . . . . . . . . . 16

3.5 Holomorphic Lie groupoids . . . . . . . . . . . . . . . . . 17

3.6 Holomorphic symplectic groupoids . . . . . . . . . . . . . . . . . . . 19

3.7 Holomorphic extension of analytic Poisson structures . . . . . . . . . . . . . 23

\section{Introduction}

Since Lie's third theorem fails for Lie algebroids, it has been a central theme of study in the theory of Lie groupoids whether a given Lie algebroid is integrable. By an integrable Lie algebroid, we mean there exists an $s$-connected and $s$-simply connected Lie groupoid of which it is the infinitesimal version. For real Lie algebroids, the integrability problem has been completely solved by Crainic-Fernandes [6] based on the work of Cattaneo-Felder on Poisson sigma models [4].

Recently, there has been increasing interest in holomorphic Lie algebroids and holomorphic Lie groupoids. It is a very natural question to find the integrability condition for holomorphic Lie algebroids. In [15], we systematically studied holomorphic Lie algebroids and their relation with real Lie algebroids. In particular, we proved that associated to any holomorphic Lie algebroid $A$, there is a canonical real Lie algebroid $A_{R}$ such that the inclusion $\mathcal{A} \rightarrow \mathcal{A}_{\infty}$ is a morphism of sheaves. Here $\mathcal{A}$ and $\mathcal{A}_{\infty}$ denote, respectively, the sheaf of holomorphic sections and the sheaf of smooth sections of $A$. In other words, a holomorphic Lie algebroid can be considered as a holomorphic vector bundle $A \rightarrow X$ whose underlying real vector bundle is endowed with a Lie algebroid structure such that, for any open subset $U \subset X$, $[\mathcal{A}(U), \mathcal{A}(U)] \subset \mathcal{A}(U)$ and the restriction of the Lie bracket $[\cdot, \cdot]$ to $\mathcal{A}(U)$ is $\mathbb{C}$-linear. It is thus natural to ask

Problem 1. Given a holomorphic Lie algebroid $A$ with underlying real Lie algebroid $A_{R}$, what is the relation between the integrability of $A$ and the integrability of $A_{R}$ ?

To tackle this problem, we need an equivalent description of holomorphic Lie algebroids. For this purpose, we will view a holomorphic Lie algebroid as a real Lie algebroid structure on a holomorphic vector bundle $A \rightarrow X$, whose almost complex structure $J_{A}: T A \rightarrow T A$ is an infinitesimal multiplicative $(1,1)$-tensor. By an infinitesimal multiplicative $(1,1)$-tensor on a (real) Lie algebroid $A$, we mean a $(1,1)$-tensor $\phi_{A}$ on $A$ such that $\phi_{A}: T A \rightarrow T A$ is an endomorphism of the tangent Lie algebroid $T A \rightarrow T X$.

On the level of Lie groupoids, if $\Gamma \rightrightarrows X$ is an $s$-connected and $s$-simply connected Lie groupoid integrating the real Lie algebroid $A$, then $J_{A}$ integrates to an automorphism $J_{\Gamma}: T \Gamma \rightarrow T \Gamma$ of 
the tangent groupoid $T \Gamma \rightrightarrows T X$ such that $J_{\Gamma}^{2}=-$ id. One can show that $J_{\Gamma}$ is fiberwise linear with respect to the vector bundle structure $T \Gamma \rightarrow \Gamma$. Hence it is a $(1,1)$-tensor on $\Gamma$. Such a $(1,1)$-tensor is called multiplicative [5, 20]. All it remains to show is that $J_{\Gamma}$ is completely integrable, i.e. $J_{\Gamma}$ is a multiplicative Nijenhuis tensor on $\Gamma$. The latter should presumably follow from the complete integrability of $J_{A}: T A \rightarrow T A$. This motivates the following:

Problem 2. Establish a one-one correspondence between infinitesimal multiplicative Nijenhuis tensors on $A$ and multiplicative Nijenhuis tensors on $\Gamma$.

To this end, we investigate multiplicative $(1,1)$-tensors on $\Gamma$ and their infinitesimal counterparts on $A$. In particular, we show that the infinitesimal of the Nijenhuis torsion of a multiplicative $(1,1)$-tensor on $\Gamma$ is the Nijenhuis torsion of its infinitesimal counterpart on $A$. We believe that our study of multiplicative $(1,1)$-tensors on a Lie groupoid will be of independent interest.

Poisson manifolds are closely related to Lie algebroids. It is well known that associated to any Poisson manifold $(X, \pi)$ there is a Lie algebroid $\left(T^{*} X\right)_{\pi}$, called the cotangent bundle Lie algebroid. If the Lie algebroid $\left(T^{*} X\right)_{\pi}$ integrates to an $s$-connected and $s$-simply connected Lie groupoid $\Gamma \rightrightarrows X$, then $\Gamma$ automatically admits a symplectic groupoid structure [17]. In this case, the Poisson structure $(X, \pi)$ is said to be integrable. See [7] (also [4]) for the integrability conditions of a smooth Poisson manifold. The same situation applies to holomorphic Poisson structures as well, and one defines integrable holomorphic Poisson manifolds in a similar fashion. As an application of our general theory, we prove that a holomorphic Poisson structure $(X, \pi)$, where $\pi=\pi_{R}+i \pi_{I}$, is integrable, if and only if $\left(X, \pi_{R}\right)$ and $\left(X, \pi_{I}\right)$ are integrable real Poisson manifolds, recovering a theorem in [5] and [20], which was proved by different methods.

The following notations are widely used in the sequel. For a manifold $M$, we use $q_{M}$ to denote the projection $T M \rightarrow M$. And given a complex manifold $X, T_{\mathbb{C}} X$ is shorthand for the complexified tangent bundle $T X \otimes \mathbb{C}$ while $T^{1,0} X$ (resp. $\left.T^{0,1} X\right)$ stands for the $+i$ - (resp. $-i$-) eigenbundle of the almost complex structure. For a Lie algebroid $A$, the Nijenhuis torsion [14, 13] of a bundle map $\phi: A \rightarrow A$ over the identity is denoted $\mathcal{N}_{\phi}$, which is a section in $\Gamma\left(\wedge^{2} A^{*} \otimes A\right)$ defined by

$$
\mathcal{N}_{\phi}(V, W)=[\phi V, \phi W]-\phi([\phi V, W]+[V, \phi W]-\phi[V, W]), \quad \forall V, W \in \Gamma(A) .
$$

When $A$ is the Lie algebroid $T X$ and $\phi: T X \rightarrow T X$ is a $(1,1)$-tensor, the Nijenhuis torsion $\mathcal{N}_{\phi}$ is a $(2,1)$-tensor on $X$.

While the paper was in writing, we learned that some similar results were also obtained independently by Cañez [2].

Acknowledgments We would like to thank Centre Émile Borel and Peking University for their hospitality while work on this project was being done. We also wish to thank Rui Fernandes and Alan Weinstein for useful discussions and comments. 


\section{Holomorphic Lie algebroids}

\subsection{Holomorphic Lie algebroids}

Holomorphic Lie algebroids were studied for various purposes in the literature. See [1, 9, 15, [12, 21] and references cited there for details.

By definition, a holomorphic Lie algebroid is a holomorphic vector bundle $A \rightarrow X$, equipped with a holomorphic bundle map $A \stackrel{\rho}{\rightarrow} T X$, called the anchor map, and a structure of sheaf of complex Lie algebras on $\mathcal{A}$, such that

(a) the anchor map $\rho$ induces a homomorphism of sheaves of complex Lie algebras from $\mathcal{A}$ to $\Theta_{X}$;

(b) and the Leibniz identity

$$
[V, f W]=(\rho(V) f) W+f[V, W]
$$

holds for all $V, W \in \mathcal{A}(U), f \in \mathcal{O}_{X}(U)$ and all open subsets $U$ of $X$.

Here $\mathcal{A}$ is the sheaf of holomorphic sections of $A \rightarrow X$ and $\Theta_{X}$ denotes the sheaf of holomorphic vector fields on $X$.

By forgetting the complex structure, a holomorphic vector bundle $A \rightarrow X$ becomes a real (smooth) vector bundle, and a holomorphic vector bundle map $\rho: A \rightarrow T X$ becomes a real (smooth) vector bundle map. Assume that $A \rightarrow X$ is a holomorphic vector bundle whose underlying real vector bundle is endowed with a Lie algebroid structure $(A, \rho,[\cdot, \cdot])$ such that, for any open subset $U \subset X$,

(a) $\quad[\mathcal{A}(U), \mathcal{A}(U)] \subset \mathcal{A}(U)$

(b) and the restriction of the Lie bracket $[\cdot, \cdot]$ to $\mathcal{A}(U)$ is $\mathbb{C}$-linear.

Then the restriction of $[\cdot, \cdot]$ and $\rho$ from $\mathcal{A}_{\infty}$ to $\mathcal{A}$ makes $A$ into a holomorphic Lie algebroid, where $\mathcal{A}_{\infty}$ denotes the sheaf of smooth sections of $A \rightarrow X$.

The following proposition states that any holomorphic Lie algebroid can be obtained out of such a real Lie algebroid in a unique way.

Proposition 2.1 ([15]). Given a structure of holomorphic Lie algebroid on the holomorphic vector bundle $A \rightarrow X$ with anchor map $A \stackrel{\rho}{\rightarrow} T X$, there exists a unique structure of real smooth Lie algebroid on the vector bundle $A \rightarrow X$ with respect to the same anchor map $\rho$ such that the inclusion of sheaves $\mathcal{A} \subset \mathcal{A}_{\infty}$ is a morphism of sheaves of real Lie algebras.

By $A_{R}$, we denote the underlying real Lie algebroid of a holomorphic Lie algebroid $A$. In the sequel, by saying that a real Lie algebroid is a holomorphic Lie algebroid, we mean that it is a holomorphic vector bundle and its Lie bracket on smooth sections restricts to a $\mathbb{C}$-linear bracket on $\mathcal{A}(U)$, for all open subset $U \subset X$. 
Assume that $(A \rightarrow X, \rho,[\cdot, \cdot])$ is a holomorphic Lie algebroid. Consider the bundle map $j: A \rightarrow A$ defining the fiberwise complex structure on $A$. It is simple to see that the Nijenhuis torsion of $j$ vanishes [15]. Hence one can define a new (real) Lie algebroid structure on $A$, denoted by $\left(A \rightarrow X, \rho_{j},[\cdot, \cdot]_{j}\right)$, where the anchor $\rho_{j}$ is $\rho \circ j$ and the bracket on $\Gamma(A)$ is given by 13 .

$$
[V, W]_{j}=[j V, W]+[V, j W]-j[V, W], \quad \forall V, W \in \Gamma(A) .
$$

In the sequel, $\left(A \rightarrow X, \rho_{j},[\cdot, \cdot]_{j}\right)$ will be called the underlying imaginary Lie algebroid and denoted by $A_{I}$. It is known that

$$
j: A_{I} \rightarrow A_{R}
$$

is a Lie algebroid isomorphism [13].

\section{$2.2 \quad$ Tangent Lie algebroids}

Recall that if $A \rightarrow M$ is a real Lie algebroid, then $T A \rightarrow T M$ is naturally a Lie algebroid, called the tangent Lie algebroid [16]. We recall its construction briefly below. For details, consult [16].

Note that for any vector bundle $E \stackrel{p}{\rightarrow} M$, the fibration $T E \stackrel{T p}{\longrightarrow} T M$ is naturally a vector bundle. Indeed, one has the double vector bundle:

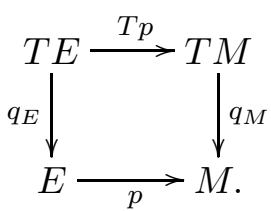

In the remainder of this section, the addition, scalar multiplication and zero section of a fiber bundle $E \stackrel{p}{\rightarrow} M$ will be denoted $+_{p}, \cdot_{p}$ and $0_{p}$ respectively. Note that the addition, scalar multiplication and zero section of $T E \stackrel{T p}{\longrightarrow} T M$ are precisely the differentials of the addition, scalar multiplication and zero section of $E \stackrel{p}{\rightarrow} M$. Hence we have

$$
\left.\frac{d}{d t} V_{t}\right|_{0}+\left._{T p} \frac{d}{d t} W_{t}\right|_{0}=\left.\frac{d}{d t}\left(V_{t}+{ }_{p} W_{t}\right)\right|_{0}
$$

if $t \mapsto V_{t}$ and $t \mapsto W_{t}$ are two paths in $E$ mapped by $p$ to the same path in $M$ so that $\left.\frac{d}{d t} V_{t}\right|_{0}$ and $\left.\frac{d}{d t} W_{t}\right|_{0}$ belong to the same fiber of $T E \stackrel{T p}{\longrightarrow} T M$. Similarly, for all $\lambda \in \mathbb{R}$, we have:

$$
\lambda \cdot T p\left(\left.\frac{d}{d t} V_{t}\right|_{0}\right)=\left.\frac{d}{d t}\left(\lambda \cdot p V_{t}\right)\right|_{0}
$$

Recall that, given a point $m \in M$, we have the canonical identification

$$
E_{m} \rightarrow T_{0_{p}(m)} E_{m}: V \mapsto \bar{V}
$$

Note that, if $V, W \in E_{m}$ and $\lambda \in \mathbb{R}$, we have

$$
\bar{V}+_{q_{E}} \bar{W}=\overline{V+{ }_{p} W}=\bar{V}+_{T p} \bar{W} \quad \text { and } \quad \lambda \cdot_{q_{E}} \bar{V}=\overline{\lambda \cdot_{p} V}=\lambda \cdot T p \bar{V}
$$


Obviously, if $V$ is a section of $E \stackrel{p}{\rightarrow} M$, then $T V$ is a section of $T E \stackrel{T p}{\rightarrow} T M$. On the other hand, the section $V \in \Gamma(E \stackrel{p}{\rightarrow} M)$ induces another section $\mathcal{S} V$ of the same bundle $T E \stackrel{T p}{\longrightarrow} T M$ defined by the relation

$$
\mathcal{S} V(v)=T 0_{p}(v)+\overline{V(m)}, \quad \text { where } v \in T_{m} M .
$$

Note that, for all $V, W \in \Gamma(E)$ and $f \in C^{\infty}(M)$, we have

$$
\mathcal{S}\left(V+{ }_{p} W\right)=\mathcal{S} V+{ }_{T p} \mathcal{S} W \quad \text { and } \quad \mathcal{S}\left(f \cdot{ }_{p} V\right)=\left(f \circ q_{M}\right) \cdot{ }_{T p} \mathcal{S} V .
$$

If $E$ is a trivialized vector bundle $M \times F \longrightarrow M$, a section is equivalent to a map $V: M \rightarrow F$. Then $\mathcal{S} V$ is the section of $T p: T M \times F \times F \longrightarrow T M$ given by

$$
T M \rightarrow T M \times F \times F: v_{m} \mapsto\left(v_{m}, 0, V(m)\right) .
$$

On the other hand, if $V \in \Gamma(E), T V$ defines another section of $T p: T E \rightarrow T M$. Under the trivialization $p: M \times F \rightarrow M, T V$ is the section

$$
T M \rightarrow T M \times F \times F: v_{m} \mapsto\left(v_{m}, V(m), v_{m}(V)\right) .
$$

It is easy to see that $\Gamma(T E \stackrel{T p}{\longrightarrow} T M)$ is generated by the sections of type $T V$ and $\mathcal{S} V$.

Taking $E=T M$ and $p=q_{M}$ in Diagram (3), we obtain the double vector bundle

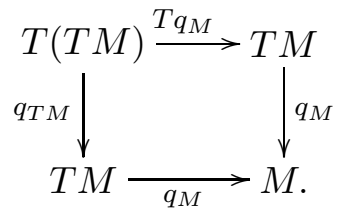

As is clearly shown by this last diagram, $T(T M)$ is endowed with two different vector bundle structures over the same base $T M$. However, there exists a canonical isomorphism of double vector bundles

$$
\sigma: T(T M) \rightarrow T(T M)
$$

called the flip map, which exchanges these two vector bundle structures $T(T M) \rightarrow T M$ :

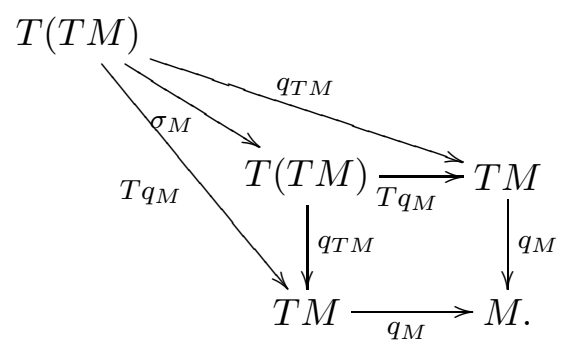

The following theorem was proved in [16].

Theorem 2.2. Let $(A \stackrel{p}{\rightarrow} M, \rho,[\cdot, \cdot])$ be a Lie algebroid. Then there exists a (unique) Lie algebroid structure on the vector bundle $T A \stackrel{T p}{\longrightarrow} T M$, with anchor $\rho_{T}:=\sigma_{\circ} \rho_{*}$, whose bracket on sections satisfies the relations

$$
[T V, T W]=T[V, W] \quad[T V, \mathcal{S} W]=\mathcal{S}[V, W] \quad[\mathcal{S} V, \mathcal{S} W]=0
$$

for any $V, W \in \Gamma(A \stackrel{p}{\rightarrow} M)$. 


\subsection{Equivalent definition of holomorphic Lie algebroids}

Proposition 2.3. Let $(A, \rho,[\cdot, \cdot])$ be a real Lie algebroid, where $A \rightarrow X$ is a holomorphic vector bundle. The following are equivalent:

(a) $(A, \rho,[\cdot, \cdot])$ is a holomorphic Lie algebroid;

(b) the map

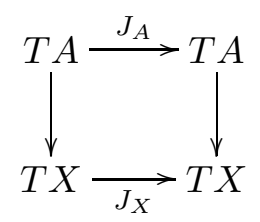

defines a Lie algebroid isomorphism, where $J_{A}$ and $J_{X}$ denote the almost complex structures on $A$ and $X$ respectively.

Proof. First of all, it is simple to check, using the local trivialization of the holomorphic bundle $A \rightarrow X$, that $\left(J_{A}, J_{X}\right)$ is indeed a bundle map. It follows from Theorem 2.2 and Eq. (44) that Diagram (5) is a Lie algebroid isomorphism if, and only if,

$$
\rho_{T} \circ J_{A}=J_{X *} \circ \rho_{T}
$$

and

$$
\left\{\begin{array}{l}
{\left[J_{A} \circ T V \circ J_{X}^{-1}, J_{A} \circ T W \circ J_{X}^{-1}\right]=J_{A} \circ[T V, T W] \circ J_{X}^{-1}} \\
{\left[J_{A} \circ T V \circ J_{X}^{-1}, J_{A} \circ \mathcal{S} W \circ J_{X}^{-1}\right]=J_{A} \circ \mathcal{S}[V, W] \circ J_{X}^{-1}} \\
{\left[J_{A} \circ \mathcal{S} V \circ J_{X}^{-1}, J_{A} \circ \mathcal{S} W \circ J_{X}^{-1}\right]=0}
\end{array}\right.
$$

for any open subset $U \subseteq X$ and all $V, W \in \mathcal{A}(U)$.

The proposition now becomes an immediate consequence of the following two observations.

(1) The anchor map $\rho: A \rightarrow T X$ is a holomorphic map if, and only if, Eq. (6) holds.

The anchor map $\rho: A \rightarrow T X$ is a holomorphic map if, and only if,

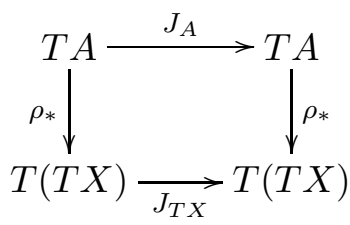

commutes, where $J_{T X}$ is the almost complex structure on $T X$ induced from the one on $X$. It is known that $J_{T X}$ is given by the following equation:

$$
J_{T X}=\sigma \circ J_{X *} \circ \sigma: T(T X) \rightarrow T(T X) .
$$

Conjugating the second horizontal line in Eq. (8) by the flip map $\sigma_{X}$, we see that Diagram (8) commutes if, and only if,

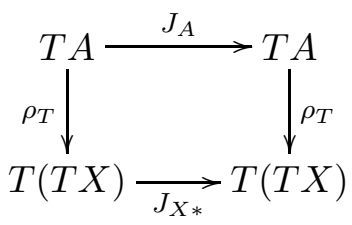


commutes, and the latter is precisely Eq. (6).

(2) The Lie bracket on $\Gamma\left(A_{U}\right)$ restricts to a $\mathbb{C}$-linear bracket on $\mathcal{A}(U)$ if, and only if, Eq. (7) is satisfied for all $V, W \in \mathcal{A}(U)$.

A smooth map between holomorphic manifolds is holomorphic if, and only if, its differential intertwines the corresponding almost complex structures. In particular, a smooth section $V \in \Gamma\left(A_{U}\right)$ is holomorphic if, and only if,

$$
J_{A} \circ T V \circ J_{X}^{-1}=T V .
$$

For all $V, W \in \mathcal{A}(U)$, we have, on the one hand,

$$
J_{A} \circ T[V, W] \circ J_{X}^{-1}=J_{A} \circ[T V, T W] \circ J_{X}^{-1}
$$

and, on the other hand according to Eq. (9),

$$
T[V, W]=[T V, T W]=\left[J_{A} \circ T V \circ J_{X}^{-1}, J_{A} \circ T W \circ J_{X}^{-1}\right] .
$$

Hence the bracket $[V, W]$ is holomorphic if, and only if,

$$
\left[J_{A} \circ T V \circ J_{X}^{-1}, J_{A} \circ T W \circ J_{X}^{-1}\right]=J_{A} \circ[T V, T W] \circ J_{X}^{-1}
$$

holds for all $V, W \in \mathcal{A}(U)$.

Secondly it is simple to check that

$$
J_{A} \circ \mathcal{S} V \circ J_{X}^{-1}=\mathcal{S}(j V) .
$$

For any $V, W \in \mathcal{A}(U)$, we have, on the one hand,

$$
\begin{aligned}
\mathcal{S}[V, j W] & =[T V, \mathcal{S}(j W)] & & \text { (by Eq. (44)) } \\
& =\left[J_{A} \circ T V \circ J_{X}^{-1}, J_{A} \circ \mathcal{S} W \circ J_{X}^{-1}\right] & & \text { (by Eqs. (9) and (10) })
\end{aligned}
$$

and, on the other hand according to Eq. (10),

$$
\mathcal{S}(j[V, W])=J_{A} \circ \mathcal{S}[V, W] \circ J_{X}^{-1} .
$$

It thus follows that $[V, j W]=j[V, W]$ if, and only if,

$$
\left[J_{A} \circ T V \circ J_{X}^{-1}, J_{A} \circ \mathcal{S} W \circ J_{X}^{-1}\right]=J_{A} \circ \mathcal{S}[V, W] \circ J_{X}^{-1}
$$

holds for all $V, W \in \mathcal{A}(U)$.

Finally, according to Eq. (9), the relation $\left[J_{A} \circ \mathcal{S} V \circ J_{X}^{-1}, J_{A} \circ \mathcal{S} W \circ J_{X}^{-1}\right]=0$ holds for any $V, W \in \mathcal{A}(U)$. 


\subsection{Holomorphic Poisson manifolds}

Definition 2.4. A holomorphic Poisson manifold is a complex manifold $X$ equipped with a holomorphic bivector field $\pi$ (i.e. $\pi \in \Gamma\left(\wedge^{2} T^{1,0} X\right)$ such that $\left.\bar{\partial} \pi=0\right)$, satisfying the equation $[\pi, \pi]=0$.

Since $\wedge^{2} T_{\mathbb{C}} X=\wedge^{2} T X \oplus i \wedge^{2} T X$, for any $\pi \in \Gamma\left(\wedge^{2} T_{\mathbb{C}} X\right)$, we can write $\pi=\pi_{R}+i \pi_{I}$, where $\pi_{R}$ and $\pi_{I} \in \Gamma\left(\wedge^{2} T X\right)$ are (real) bivector fields on $X$. The following result was proved in [15].

Theorem 2.5 ([15]). Given a complex manifold $X$ with associated almost complex structure $J$, the following are equivalent:

(a) $\quad \pi=\pi_{R}+i \pi_{I} \in \Gamma\left(\wedge^{2} T^{1,0} X\right)$ is a holomorphic Poisson bivector field;

(b) $\quad\left(\pi_{I}, J\right)$ is a Poisson Nijenhuis structure on $X$ and $\pi_{R}^{\sharp}=\pi_{I}^{\sharp} \circ J^{*}$;

As a consequence, both $\pi_{R}$ and $\pi_{I}$ are Poisson tensors and they constitute a biHamiltonian system [14]. Both $\pi_{R}$ and $\pi_{I}$ define brackets $\{\cdot, \cdot\}_{R}$ and $\{\cdot, \cdot\}_{I}$ on $C^{\infty}(M, \mathbb{R})$ in the standard way. These extend to $C^{\infty}(M, \mathbb{C})$ by $\mathbb{C}$-linearity. In a local chart $\left(z_{1}=x_{1}+i y_{1}, \cdots, z_{n}=\right.$ $\left.x_{n}+i y_{n}\right)$ of complex coordinates of $X$, we indeed have

$$
\begin{array}{ll}
\left\{x_{i}, x_{j}\right\}_{R}=\frac{1}{4} \Re\left\{z_{i}, z_{j}\right\}, & \left\{x_{i}, x_{j}\right\}_{I}=\frac{1}{4} \Im\left\{z_{i}, z_{j}\right\}, \\
\left\{y_{i}, y_{j}\right\}_{R}=-\frac{1}{4} \Re\left\{z_{i}, z_{j}\right\}, & \left\{y_{i}, y_{j}\right\}_{I}=-\frac{1}{4} \Im\left\{z_{i}, z_{j}\right\}, \\
\left\{x_{i}, y_{j}\right\}_{R}=\frac{1}{4} \Im\left\{z_{i}, z_{j}\right\}, & \left\{x_{i}, y_{j}\right\}_{I}=-\frac{1}{4} \Re\left\{z_{i}, z_{j}\right\} .
\end{array}
$$

Here $\Re$ and $\Im$ denote the real and imaginary parts of a complex number.

Now we consider the cotangent bundle Lie algebroid of a holomorphic Poisson manifold and identify its associated real and imaginary Lie algebroids. Assume that $(X, \pi)$ is a holomorphic Poisson manifold, where $\pi=\pi_{R}+i \pi_{I} \in \Gamma\left(\wedge^{2} T^{1,0} X\right)$. Let $A=\left(T^{*} X\right)_{\pi}$ be its corresponding holomorphic Lie algebroid, which can be defined in a similar way as in the smooth case. To be more precise, let $\Phi$ and $\Psi$, respectively, be the holomorphic bundle maps

$$
\Phi: T X \rightarrow T^{1,0} X, \Phi=\frac{1}{2}(1-i J)
$$

and

$$
\Psi: T^{*} X \rightarrow\left(T^{1,0} X\right)^{*}, \Psi=1-i J^{*},
$$

where $J$ is the almost complex structure on $X$. Define the anchor $\rho:\left(T^{*} X\right)_{\pi} \rightarrow T X$ to be $\rho=\Phi^{-1} \circ \pi^{\#} \circ \Psi$ and the bracket

$$
[\alpha, \beta]_{\pi}=L_{\rho \alpha} \beta-L_{\rho \beta} \alpha-\mathrm{d}(\rho \alpha, \beta)
$$

$\forall \alpha, \beta \in \Gamma\left(\left.T^{*} X\right|_{U}\right)$ holomorphic. One easily sees that $\left(T^{*} X\right)_{\pi}$ is a holomorphic Lie algebroid. Next proposition describes its real and imaginary Lie algebroids.

Proposition $2.6([15])$. Let $(X, \pi)$ be a holomorphic Poisson manifold, where $\pi=\pi_{R}+i \pi_{I} \in$ $\Gamma\left(\wedge^{2} T^{1,0} X\right)$. Then the underlying real and imaginary Lie algebroids of $\left(T^{*} X\right)_{\pi}$ are isomorphic to $\left(T^{*} X\right)_{4 \pi_{R}}$ and $\left(T^{*} X\right)_{4 \pi_{I}}$, respectively. 


\section{$3 \quad$ Holomorphic Lie groupoids and holomorphic symplectic groupoids}

\subsection{Multiplicative (1,1)-tensors on Lie groupoids}

Recall that a skew-symmetric $(k, 1)$-tensor on a smooth manifold $M$ can be seen either as a section of the vector bundle $\wedge^{k} T^{*} M \otimes T M \rightarrow M$, or as a bundle map $\wedge^{k} T M \rightarrow T M$ over the identity on $M$. If $\Gamma \rightrightarrows M$ is a Lie groupoid, then $T \Gamma \rightrightarrows T M$ is naturally a Lie groupoid, called the tangent Lie groupoid [16]. Indeed for any $k \geq 1$, this Lie groupoid structure extends naturally to a Lie groupoid $\wedge^{k} T \Gamma \rightrightarrows \wedge^{k} T M$, whose source, target, inverse and product maps are given by $\wedge^{k} T s, \wedge{ }^{k} T t, \wedge^{k} T \iota$ and $\wedge^{k} T m$, respectively. Here $s, t, \iota$ and $m$ are the source, target, inverse and product maps of the groupoid $\Gamma \rightrightarrows M$.

Definition 3.1. A multiplicative $(k, 1)$-tensor $\phi$ on a Lie groupoid $\Gamma \rightrightarrows M$ is a pair $\left(\phi_{\Gamma}, \phi_{M}\right)$ of $(k, 1)$-tensors on $\Gamma$ and $M$ respectively such that

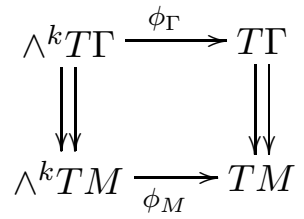

is a Lie groupoid morphism.

Remark 3.2. It is simple to see that if $\left(\phi_{\Gamma}, \phi_{M}\right)$ is a multiplicative $(k, 1)$-tensor, $\phi_{M}$ is completely determined by $\phi_{\Gamma}$, which is the restriction of $\phi_{\Gamma}$ to the unit space $M$. Hence we often use $\phi_{\Gamma}$ to denote a multiplicative $(k, 1)$-tensor.

Proposition 3.3. If $\left(\phi_{\Gamma}, \phi_{M}\right)$ is a multiplicative $(1,1)$-tensor on a Lie groupoid $\Gamma \rightrightarrows M$, then $\left(\mathcal{N}_{\phi_{\Gamma}}, \mathcal{N}_{\phi_{M}}\right)$ is a multiplicative $(2,1)$-tensor on $\Gamma$.

The proof requires two lemmas. The first one, a general fact about Nijenhuis tensors, is a straightforward consequence of Eq. (1). A $(k, 1)$-tensor $\phi$ on a manifold $N$ is said to be tangent to a submanifold $S \subset N$ if $\phi$ maps $\wedge^{k} T S$ to $T S$. Any $(k, 1)$-tensor $\phi$ tangent to $S$ induces by restriction a $(k, 1)$-tensor on the submanifold $S$.

The following lemma can be easily verified.

Lemma 3.4. If $S \subset N$ is a submanifold, and $\phi$ is $(1,1)$-tensor tangent to $S$, then $\mathcal{N}_{\phi}$ is tangent to $S$. Moreover the restriction of $\mathcal{N}_{\phi}$ to $S$ is the Nijenhuis tensor of the restriction of $\phi$ to $S$.

The second lemma is a general fact regarding Lie groupoids, the proof of which is left to the reader. Recall that the graph of the multiplication of a given Lie groupoid $\Gamma \rightrightarrows M$ is the submanifold $\Lambda_{\Gamma}$

$$
\Lambda_{\Gamma}=\left\{\left(\gamma_{1}, \gamma_{2}, \gamma_{1} \gamma_{2}\right) \mid \forall \gamma_{1}, \gamma_{2} \in \Gamma \text { s.t. } t\left(\gamma_{1}\right)=s\left(\gamma_{2}\right)\right\}
$$

of $\Gamma \times \Gamma \times \Gamma$. Given a map $\Psi: R \rightarrow R^{\prime}$, we denote by $\Psi^{(3)}$ the map

$$
\Psi^{(3)}: R \times R \times R \rightarrow R^{\prime} \times R^{\prime} \times R^{\prime}:(x, y, z) \mapsto(\Psi(x), \Psi(y), \Psi(z)) .
$$


Lemma 3.5. (a) The graphs of the multiplications of the Lie groupoids $\Gamma \rightrightarrows M, T \Gamma \rightrightarrows T M$ and $\wedge^{2} T \Gamma \rightrightarrows \wedge^{2} T M$ are related as follows

$$
\Lambda_{T \Gamma}=T \Lambda_{\Gamma} \quad \Lambda_{\wedge^{2} T \Gamma}=\wedge^{2} T \Lambda_{\Gamma}
$$

(b) A smooth map $\Psi$ from a Lie groupoid $\Gamma \rightrightarrows M$ to another Lie groupoid $\Gamma^{\prime} \rightrightarrows M^{\prime}$ is a groupoid homomorphism if, and only if, $\Psi^{(3)}$ maps $\Lambda_{\Gamma}$ to $\Lambda_{\Gamma^{\prime}}$.

Proof of Proposition 3.3. By Lemma 3.5(b) , since the pair $\left(\phi_{\Gamma}, \phi_{M}\right)$ is a Lie groupoid homomorphism, $\phi_{\Gamma}^{(3)}: T \Gamma^{3} \rightarrow T \Gamma^{3}$ maps $\Lambda_{T \Gamma}$ to itself. By Lemma 3.5((a) , the latter is isomorphic to $T \Lambda_{\Gamma}$. Hence $\phi_{\Gamma}^{(3)}$ is tangent to $\Lambda_{\Gamma}$.

By Lemma 3.4, it follows that $\mathcal{N}_{\phi_{\Gamma}^{(3)}}$ is also tangent to $\Lambda_{\Gamma}$. A simple computation yields that

$$
\mathcal{N}_{\phi_{\Gamma}^{(3)}}=\mathcal{N}_{\phi_{\Gamma}}^{(3)}
$$

Therefore $\mathcal{N}_{\phi_{\Gamma}}^{(3)}$ maps $\wedge^{2} T \Lambda_{\Gamma}$ to $T \Lambda_{\Gamma}$. According to Lemma 3.5 again, this amounts to saying that $\left(\mathcal{N}_{\phi_{\Gamma}}, \mathcal{N}_{\phi_{M}}\right)$ is indeed a Lie groupoid homomorphism.

\subsection{Nijenhuis torsion of multiplicative $(1,1)$-tensors}

Recall that, for any vector bundle $E \stackrel{p}{\rightarrow} M$, one has the double vector bundle (3). Similarly, one has the double vector bundle

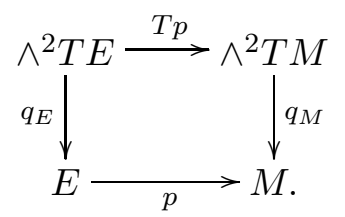

In particular, when $E$ is the tangent bundle $T M \stackrel{p}{\rightarrow} M$, we have the double bundles

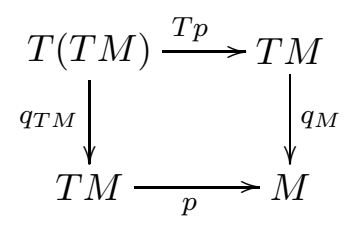

and

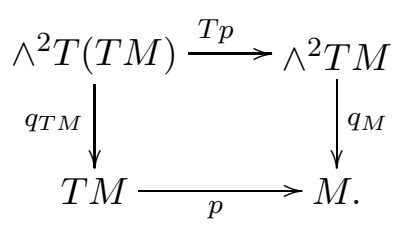


There is an extension of the canonical flip map $\sigma: T(T M) \rightarrow T(T M)$, denoted by $\sigma^{(1)}$ in this section, to $T\left(\wedge^{2} T M\right)$, which is defined as follows. Let $\mu \in T_{e_{1} \wedge e_{2}}\left(\wedge^{2} T M\right)$ be any tangent vector, where $e_{1}, e_{2} \in T_{m} M$. Write

$$
\mu=\left.\frac{d}{d t} e_{1}(t) \wedge e_{2}(t)\right|_{0}
$$

where $e_{1}(t), e_{2}(t) \in T_{m(t)} M$. Define

$$
\sigma^{(2)}(\mu)=\left.\left.\sigma^{(1)} \frac{d}{d t} e_{1}(t)\right|_{0} \wedge \sigma^{(1)} \frac{d}{d t} e_{2}(t)\right|_{0} .
$$

Then $\sigma^{(2)}(\mu)$ is a vector in $\wedge^{2} T_{v}(T M)$. Hence $\sigma^{(2)}$ maps $T_{e_{1} \wedge e_{2}}\left(\wedge^{2} T M\right)$ to $\wedge^{2} T_{v}(T M)$, where $v=(T p)\left(e_{1} \wedge e_{2}\right) \in T_{m} M$. Note that $v$ changes according to $e_{1} \wedge e_{2}$.

Lemma 3.6. (a) $\sigma^{(1)}$ is an isomorphism of double vector bundle (12), which interchanges the horizontal and vertical bundle structures and induces the identities on the side bundles.

(b) $\sigma^{(2)}$ is an isomorphism from the double vector bundle

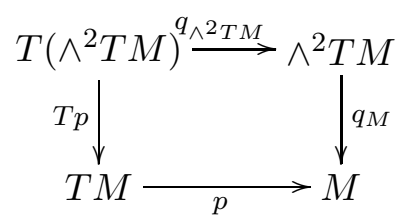

to the double vector bundle (13), which induces the identities on the side bundles.

Let $\phi: T M \rightarrow T M$ be a $(1,1)$-tensor on $M$. Then $T \phi$ is a morphism of horizontal vector bundle $T(T M) \stackrel{T p}{\rightarrow} T M$ to itself over the identity. Define

$$
\mathbb{T} \phi=\sigma^{(1)} \circ T \phi \circ\left(\sigma^{(1)}\right)^{-1} .
$$

From Lemma 3.6, it follows that $\mathbb{T} \phi$ is a morphism of vertical vector bundle $q_{T M}: T(T M) \rightarrow$ $T M$ to itself over the identity.

Similarly, if $\phi: \wedge^{2} T M \rightarrow T M$ is a $(2,1)$-tensor on $M$, then

$$
\mathbb{T} \phi=\sigma^{(1)} \circ T \phi \circ\left(\sigma^{(2)}\right)^{-1}
$$

is a morphism from the vector bundle $q_{T M}: \wedge^{2} T(T M) \rightarrow T M$ to the vector bundle $q_{T M}$ : $T(T M) \rightarrow T M$ over the identity.

Proposition 3.7. If $\phi$ is a $(1,1)$-tensor (resp. $(2,1)$-tensor) on a manifold $M$, then $\mathbb{T} \phi$ is a (1,1)-tensor (resp. $(2,1)$-tensor) on the manifold TM. Moreover, we have

$$
\mathcal{N}_{\mathbb{T} \phi}=\mathbb{T} \mathcal{N}_{\phi}
$$


Proof. It remains to prove Eq. (14). Note that for any section $V$ of $p: T M \rightarrow M, T V$ is a section of $T p: T(T M) \rightarrow T M$, which is a tangent Lie algebroid. Let

$$
\mathbb{T} V=\sigma^{(1)} \circ T V .
$$

Then $\mathbb{T} V$ is a section of the bundle $q_{T M}: T(T M) \rightarrow T M$, i.e. a vector field on $T M$. Considering $p: T M \rightarrow M$ as a Lie algebroid and $T p: T(T M) \rightarrow T M$ as its tangent Lie algebroid, according to Eq. (4), we have $[T V, T W]=T[V, W]$, where the left hand side of the equation refers to the Lie bracket of the tangent Lie algebroid $T(T M) \rightarrow T M$. Hence

$$
\mathbb{T}[V, W]=\sigma^{(1)} \circ T[V, W]=\sigma^{(1)} \circ[T V, T W]=[\mathbb{T} V, \mathbb{T} W] .
$$

Here the bracket $[\mathbb{T} V, \mathbb{T} W]$ on the right hand side refers to the bracket on the vector fields $\mathfrak{X}(T M)$, and the last equality follows from the definition of tangent Lie algebroid.

Now if $\phi$ is a $(1,1)$-tensor on $M$, we have

$$
(\mathbb{T} \phi)(\mathbb{T} V)=\sigma^{(1)} \circ T \phi \circ\left(\sigma^{(1)}\right)^{-1} \circ \sigma^{(1)} \circ T V=\sigma^{(1)} \circ T \phi \circ T V=\sigma^{(1)} \circ T \phi(V)=\mathbb{T}(\phi V) .
$$

Similarly, for any $(2,1)$-tensor $\psi$ on $M$ and any sections $V, W$ of $T M \rightarrow M$, we have

$$
(\mathbb{T} \psi)(\mathbb{T} V, \mathbb{T} W)=\mathbb{T}(\psi(V, W)) .
$$

Using Eqs. (15) and (17), a simple computation leads to

$$
\mathcal{N}_{\mathbb{T} \phi}(\mathbb{T} V, \mathbb{T} W)=\left(\mathbb{T} \mathcal{N}_{\phi}\right)(\mathbb{T} V, \mathbb{T} W) .
$$

On the other hand, using local coordinates, it is easy to see that for any $u \in T M$ with $u \neq 0$ and $v \in T_{u}(T M)$, there always exists a section $V$ of $T M \rightarrow M$ such that $\mathbb{T} V$ passes through $v$ at $u$. Since both $\mathcal{N}_{\mathbb{T} \phi}$ and $\mathbb{T} \mathcal{N}_{\phi}$ are $(2,1)$-tensors on $T M$, it follows that they coincide at all points of $T M$ except for the zero section of $T M$. Hence they must be equal at all points by continuity.

Now let $\Gamma \rightrightarrows M$ be a Lie groupoid with Lie algebroid $A$. By definition $A$ is identified with the subbundle $T_{M}^{s} \Gamma$ of $\left.T \Gamma\right|_{M}$. In the sequel, we use this identification implicitly.

Let $\left(\phi_{\Gamma}, \phi_{M}\right)$ be a multiplicative $(1,1)$-tensor on $\Gamma$. Then by Proposition 3.7, $\mathbb{T} \phi_{\Gamma}$ is a $(1,1)$ tensor on the manifold $T \Gamma$. Similarly if $\left(\psi_{\Gamma}, \psi_{M}\right)$ is a multiplicative $(2,1)$-tensor on $\Gamma$, then $\mathbb{T} \psi_{\Gamma}$ is a $(2,1)$-tensor on the manifold $T \Gamma$.

Lemma 3.8. Let $\left(\phi_{\Gamma}, \phi_{M}\right)$ and $\left(\psi_{\Gamma}, \psi_{M}\right)$ be a multiplicative $(1,1)$-tensor and $(2,1)$-tensor on a Lie groupoid $\Gamma \rightrightarrows M$, respectively. Then both $\mathbb{T} \phi_{\Gamma}$ and $\mathbb{T} \psi_{\Gamma}$ are tangent to the submanifold $T_{M}^{s} \Gamma \subset T \Gamma$. Hence they define a $(1,1)$-tensor and $(2,1)$-tensor on the submanifold $T_{M}^{s} \Gamma$ of $T \Gamma$.

Proof. By definition, $\mathbb{T} \phi_{\Gamma}=\sigma^{(1)} \circ T \phi \circ\left(\sigma^{(1)}\right)^{-1}$. It is well-known that $\sigma^{(1)}: T_{T M}^{T s} T \Gamma \rightarrow T\left(T_{M}^{s} \Gamma\right)$ is an isomorphism. Since $\phi: T \Gamma \rightarrow T \Gamma$ is a groupoid morphism of the tangent groupoid $T \Gamma \rightrightarrows T M$, it follows that $T_{T M}^{T s} T \Gamma$ is stable under $T \phi$. Hence it follows that $T\left(T_{M}^{s} \Gamma\right)$ is stable under $\mathbb{T} \phi$. That is, $\mathbb{T} \phi_{\Gamma}$ is tangent to the submanifold $T_{M}^{s} \Gamma \subset T \Gamma$.

Similarly, one proves that $\mathbb{T} \psi_{\Gamma}$ is also tangent to $T_{M}^{s} \Gamma$. 
Now we introduce

Definition 3.9. If $\left(\phi_{\Gamma}, \phi_{M}\right)$ (resp. $\left.\left(\psi_{\Gamma}, \psi_{M}\right)\right)$ is a multiplicative $(1,1)$-tensor (resp. $(2,1)$ tensor) on $\Gamma$, we define $\mathbb{L i e} \phi_{\Gamma}\left(\right.$ resp. $\left.\mathbb{L i e} \psi_{\Gamma}\right)$ to be the restriction of $\mathbb{T} \phi_{\Gamma}$ (resp. $\left.\mathbb{T} \psi_{\Gamma}\right)$ to $A$ (being identified with $T_{M}^{s} \Gamma$ and considered as a submanifold of $T \Gamma$ ).

The main result of this section is the following:

Theorem 3.10. Let $\Gamma \rightrightarrows M$ be a Lie groupoid with Lie algebroid $A$. If $\phi_{\Gamma}$ is a multiplicative $(1,1)$-tensor on $\Gamma$, then $\mathbb{L i e}\left(\phi_{\Gamma}\right)$ is a $(1,1)$-tensor on $A$ (as a manifold), and $\operatorname{Lie}\left(\mathcal{N}_{\phi_{\Gamma}}\right)$ is a $(2,1)$-tensor on $A$ (as a manifold). Moreover we have

$$
\mathcal{N}_{\mathbb{L i e}\left(\phi_{\Gamma}\right)}=\operatorname{Lie}\left(\mathcal{N}_{\phi_{\Gamma}}\right) .
$$

Proof. In Lemma 3.4, taking $N=T \Gamma$ and $S=T_{M}^{s} \Gamma$, we obtain that $\mathcal{N}_{\mathbb{L i e} \phi_{\Gamma}}=\left.\mathcal{N}_{\mathbb{T} \phi_{\Gamma}}\right|_{T_{M}^{s} \Gamma \text {. The }}$ latter is equal to $\left.\mathbb{T} \mathcal{N}_{\phi_{\Gamma}}\right|_{T_{M}^{s} \Gamma}$ according to Proposition 3.7, which is $\operatorname{Lie}\left(\mathcal{N}_{\phi_{\Gamma}}\right)$ by definition.

\subsection{Infinitesimal multiplicative $(1,1)$-tensors}

Definition 3.11. Let $(A, \rho,[\cdot, \cdot])$ be a real Lie algebroid. An infinitesimal multiplicative $(1,1)$ tensor on $A$ is a pair $\left(\phi_{A}, \phi_{M}\right)$ of $(1,1)$-tensors on $A$ and $M$ such that the following diagram

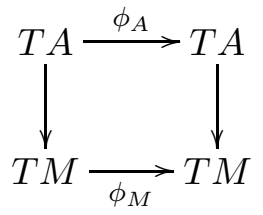

is a Lie algebroid homomorphism, where $T A \rightarrow T M$ is the tangent Lie algebroid of $A \rightarrow M$.

Remark 3.12. It is simple to see that $\phi_{M}$ is completely determined by $\phi_{A}$, which is equal to the restriction of $\phi_{A}$ to the zero section $M \subset A$. In the sequel, we sometimes simply use $\phi_{A}$ to denote an infinitesimal multiplicative $(1,1)$-tensor on $A$.

In particular, for any holomorphic Lie algebroid $A \rightarrow X$, the almost complex structures $\left(J_{A}, J_{X}\right)$ is an infinitesimal multiplicative $(1,1)$-tensor on $A$ according to Proposition 2.3 .

Let $\left(\phi_{\Gamma}, \phi_{M}\right)$ be a multiplicative $(1,1)$-tensor on a Lie groupoid $\Gamma \rightrightarrows M$. According to Theorem 3.10, $\operatorname{Lie}\left(\phi_{\Gamma}\right)$ is a $(1,1)$-tensor on $A$. Moreover, by construction, it is clear that $\operatorname{Lie}\left(\phi_{\Gamma}\right)$ is a homomorphism of the tangent Lie algebroid $T A \rightarrow T M$ to itself. Hence the assignment

$$
\phi_{\Gamma} \stackrel{\text { Lie }}{\longrightarrow} \phi_{A}
$$

is a map from the space of multiplicative $(1,1)$-tensors on $\Gamma \rightrightarrows M$ to the space of infinitesimal multiplicative $(1,1)$-tensors on $A$. The next proposition indicates that this map is indeed a bijection when $\Gamma$ is s-connected and s-simply connected.

Proposition 3.13. Let $\Gamma \rightrightarrows M$ be a s-connected and s-simply connected Lie groupoid with Lie algebroid $(A, \rho,[\cdot, \cdot])$. The assignment Lie as in Eq. (20) is a bijection between multiplicative $(1,1)$-tensors on $\Gamma \rightrightarrows M$ and infinitesimal multiplicative $(1,1)$-tensors on $A$. 
Proof. Let $\left(\phi_{A}, \phi_{M}\right)$ be an infinitesimal multiplicative $(1,1)$-tensor on $A$. Since the tangent Lie groupoid $T \Gamma \rightrightarrows T M$ is s-connected and s-simply connected, there exists a Lie groupoid homomorphism $\left(\phi_{\Gamma}, \phi_{M}\right)$ :

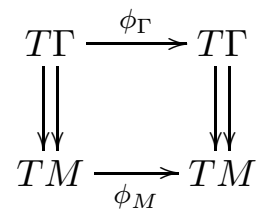

which integrates the Lie algebroid morphism (19). It remains to show that $\phi_{\Gamma}$ is a $(1,1)$-tensor on $\Gamma$.

Since $\phi_{A}: T A \rightarrow T A$ is a $(1,1)$-tensor, we have the following commutative diagram of Lie algebroid morphisms:

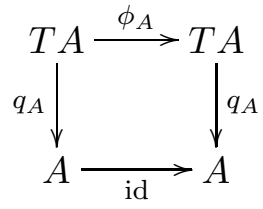

which implies the following commutative diagram of Lie groupoid homomorphisms:

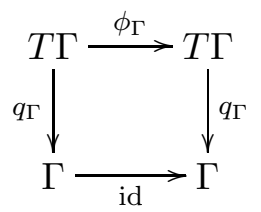

Hence, $\phi_{\Gamma}: T \Gamma \rightarrow T \Gamma$ is a map over the identity of $\Gamma$. It remains to check that it is fiberwise linear.

We recall some well-known constructions in [17]. Note that there is a natural Lie groupoid structure on $T \Gamma \times_{\Gamma} T \Gamma \rightrightarrows T M \times_{M} T M$, and the fiberwise addition map:

$$
+_{\Gamma}(V, W)=V+W \quad \forall V, W \in T_{\gamma} \Gamma, \gamma \in \Gamma
$$

is a Lie groupoid homomorphism from $T \Gamma \times_{\Gamma} T \Gamma \rightrightarrows T M \times_{M} T M$ to $T \Gamma \rightrightarrows T M$. On the level of Lie algebroids, there is a natural Lie algebroid structure on the vector bundle $T A \times{ }_{A} T A \rightarrow T M \times{ }_{M} T M$, and the fiberwise addition map:

$$
+_{A}(V, W)=V+W \quad \forall V, W \in T_{a} A, a \in A
$$

is a Lie algebroid morphism from $T A \times_{A} T A \rightarrow T M \times_{M} T M$ to $T A \rightarrow T M$. The Lie groupoid homomorphism $\phi_{\Gamma}$ induces a Lie groupoid homomorphism $\phi_{\Gamma} \times \phi_{\Gamma}$ from $T \Gamma \times_{\Gamma} T \Gamma \rightrightarrows$ $T M \times_{M} T M$ to itself, while the Lie algebroid morphism $\phi_{A}$ induces a Lie algebroid morphism $\phi_{A} \times \phi_{A}$ from $T A \times_{A} T A \rightarrow T M \times_{M} T M$ to itself.

Moreover, we have

$$
\left\{\begin{array}{l}
\operatorname{Lie}\left(T \Gamma \times_{\Gamma} T \Gamma\right)=T A \times_{A} T A \\
\mathbb{L i e}\left(\phi_{\Gamma} \times \phi_{\Gamma}\right)=\phi_{A} \times \phi_{A} \\
\mathbb{L i e}\left(+_{\Gamma}\right)=+_{A}
\end{array}\right.
$$


Since

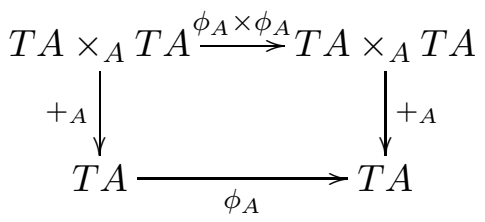

is a commutative diagram of Lie algebroid morphisms,

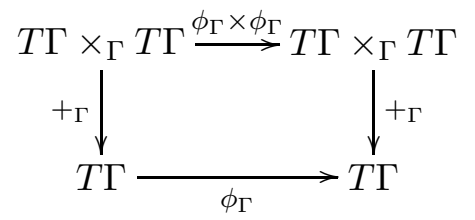

is a commutative diagram of Lie groupoid homomorphisms. Therefore the map $\phi_{\Gamma}: T \Gamma \rightarrow T \Gamma$ respects the fiberwise addition of the vector bundle $q_{\Gamma}: T \Gamma \rightarrow \Gamma$. Since $\phi_{\Gamma}$ is a smooth map, it follows that $\phi_{\Gamma}$ must be fiberwise linear. Hence, it is a multiplicative $(1,1)$-tensor on $\Gamma$.

Finally, it is simple to see, from the construction, that

$$
\operatorname{Lie}\left(\phi_{\Gamma}\right)=\phi_{A} .
$$

This concludes the proof.

\subsection{Multiplicative Nijenhuis tensors on Lie groupoids}

We now can state one of the main theorems of this section.

Theorem 3.14. Let $\Gamma \rightrightarrows M$ be a s-connected and s-simply connected Lie groupoid with Lie algebroid $(A, \rho,[\cdot, \cdot])$. The assignment $\mathbb{L}$ ie as in Eq. (20) is a bijection between multiplicative Nijenhuis tensors on $\Gamma \rightrightarrows M$ and infinitesimal multiplicative Nijenhuis tensors on $A$.

Proof. Theorem 3.10 implies that

$$
\operatorname{Lie}\left(\mathcal{N}_{\phi_{\Gamma}}\right)=\mathcal{N}_{\operatorname{Lie}\left(\phi_{\Gamma}\right)}=\mathcal{N}_{\phi_{A}} .
$$

Since $\phi_{A}: T A \rightarrow T A$ is a Nijenhuis tensor, the Nijenhuis torsion $\mathcal{N}_{\phi_{A}}$ vanishes. The latter is equivalent to the commutativity of the diagram

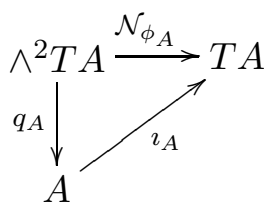

where $\imath_{A}: A \hookrightarrow T A$ is the embedding of the zero section. Since all the maps in the above diagram are Lie algebroid morphisms, it follows from the relations $q_{A}=\operatorname{Lie}\left(q_{\Gamma}\right)$ and $\imath_{A}=$ $\operatorname{Lie}\left(\imath_{\Gamma}\right)$ that one has a commutative diagram of Lie groupoid homomorphisms

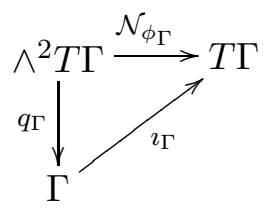


where $\imath_{\Gamma}: \Gamma \rightarrow T \Gamma$ is the embedding to the zero section. Therefore the Nijenhuis torsion $\mathcal{N}_{\phi_{\Gamma}}$ vanishes. This completes the proof of the theorem.

\subsection{Holomorphic Lie groupoids}

Definition 3.15. : A holomorphic Lie groupoid is a (smooth) Lie groupoid $\Gamma \rightrightarrows X$, where both $\Gamma$ and $X$ are complex manifolds and all the structure maps $m, \epsilon, \iota, s$ and $t$ are holomorphic maps.

This definition requires a justification. The manifold

$$
\Gamma_{2}=\left\{\left(\gamma_{1}, \gamma_{2}\right) \mid t\left(\gamma_{1}\right)=s\left(\gamma_{2}\right), \gamma_{1}, \gamma_{2} \in \Gamma\right\}
$$

admits a natural complex manifold structure since both the source and target maps are holomorphic surjective submersions. It thus makes sense to require the multiplication map to be holomorphic.

The following result essentially follows from the definition and the Newlander-Nirenberg theorem.

Proposition 3.16. The following assertions are equivalent:

(a) $\Gamma \rightrightarrows X$ is a holomorphic Lie groupoid;

(b) $\Gamma \rightrightarrows X$ is a real Lie groupoid, where $\Gamma$ and $X$ are almost complex manifolds with associated almost complex structures $J_{\Gamma}$ and $J_{X}$ respectively such that $\left(J_{\Gamma}, J_{X}\right)$ is a multiplicative Nijenhuis tensor on $\Gamma \rightrightarrows X$.

A holomorphic Lie algebroid is said to be integrable if it is the Lie algebroid associated to some holomorphic Lie groupoid.

The main theorem of this section is the following

Theorem 3.17. Assume that $A \rightarrow X$ is a holomorphic Lie algebroid. Let $\Gamma$ be a s-connected and s-simply connected Lie groupoid integrating the underlying real Lie algebroid $A_{R}$. Then $\Gamma$ admits a natural complex structure which makes it into a holomorphic Lie groupoid with its holomorphic Lie algebroid being $A \rightarrow X$.

In other words, a holomorphic Lie algebroid $A$ is integrable if, and only if, its underlying real Lie algebroid $A_{R}$ is integrable. Similarly, a holomorphic Lie algebroid is integrable if, and only if, its underlying imaginary Lie algebroid $A_{I}$ is integrable.

Proof. According to Proposition 2.3, the almost complex structure $\left(J_{A}, J_{X}\right)$ is an infinitesimal multiplicative Nijenhuis tensor on $A$. By Theorem 3.14, it integrates to a multiplicative Nijenhuis tensor $\left(J_{\Gamma}, J_{X}\right)$ on $\Gamma \rightrightarrows X$. Since $-\mathrm{id}_{\Gamma}: T \Gamma \rightarrow T \Gamma$ is also a multiplicative $(1,1)$ tensor and $\operatorname{Lie}\left(-\mathrm{id}_{\Gamma}\right)=-\mathrm{id}_{A}$, it follows that $J_{\Gamma}^{2}=-\mathrm{id}_{\Gamma}$ and therefore $J_{\Gamma}$ is an almost complex structure on $\Gamma$. The conclusion thus follows from Proposition 3.16 . 
As a consequence, we can determine whether a holomorphic Lie algebroid is integrable by applying the integrability criteria of Crainic-Fernandes: Theorem 4.1 in [6] to its underlying real Lie algebroid.

Remark 3.18. Note that, a complex structure on the Lie algebra of a real Lie group G extends uniquely to an integrable complex Lie group structure on $G$. This is false for groupoids. The condition of $s$-connectedness and s-simply connectedness is indeed necessary. For instance, take a bundle of groups $X \times \mathbb{C} \rightrightarrows X$ and consider it as a groupoid, where $X$ is a complex manifold and $\mathbb{C}$ is equipped with the additive group structure. Let $L$ be a smooth non-holomorphic function from $X$ to the space of lattices in $\mathbb{C}$ (for instance $X=\mathbb{C}$ and $f(x+i y)=1+i \sin (x))$ and consider the quotient groupoid $(X \times \mathbb{C}) / L \rightrightarrows X$. Clearly this is a real Lie groupoid integrating the underlying real Lie algebroid $X \times \mathbb{C} \rightarrow X$ with zero anchor and zero bracket. However the holomorphic structure on the Lie algebroid does not extend to a holomorphic groupoid structure on this quotient groupoid $(X \times \mathbb{C}) / L \rightrightarrows X$.

A holomorphic Lie algebroid may not be always integrable, as shown in the following

Example 3.19. Let $X$ be a complex manifold and $\omega$ a holomorphic closed 2-form on $X$. Then $A: T X \oplus(X \times \mathbb{C}) \rightarrow X$ is naturally equipped with a holomorphic Lie algebroid structure, where the anchor is the projection onto the first component, and the Lie bracket is

$$
[(X, f),(Y, g)]=([X, Y], X(g)-Y(f)+\omega(X, Y)),
$$

$\forall X, Y \in \mathcal{A}(U), f, g \in \mathcal{O}_{X}(U)$. It is simple to see that its underlying real Lie algebroid $A_{R}$ is isomorphic to $T X \oplus\left(X \times \mathbb{R}^{2}\right) \rightarrow X$, where the Lie bracket is given by

$$
\left[\left(X, f_{1}, f_{2}\right),\left(Y, g_{1}, g_{2}\right)\right]=\left([X, Y], X\left(g_{1}\right)-Y\left(f_{1}\right)+\omega_{1}(X, Y), X\left(g_{2}\right)-Y\left(f_{2}\right)+\omega_{2}(X, Y)\right),
$$

$\forall X, Y \in \mathfrak{X}(X)$ and $f_{1}, f_{2}, g_{1}, g_{2} \in C^{\infty}\left(\mathbb{R}^{2}, \mathbb{R}\right)$, where $\omega_{1}$ and $\omega_{2}$ are the real and imaginary parts of $\omega$, respectively, i.e. $\omega=\omega_{1}+i \omega_{2}$.

According to Example 3.7 in [6], the Lie algebroid $T X \oplus\left(X \times \mathbb{R}^{2}\right) \rightarrow X$ is integrable if and only if the group of periods of $\left(\omega_{1}, \omega_{2}\right)$ :

$$
\left\{\left(\int_{\gamma} \omega_{1}, \int_{\gamma} \omega_{2}\right) \mid[\gamma] \in \pi_{2}(M, x)\right\}
$$

is a discrete subgroup of $\mathbb{R}^{2}$ (the argument in [6] is only presented for $\mathbb{R}$-valued closed 2-forms, but it clearly extends to $\mathbb{R}^{2}$-valued closed 2 -forms).

Consider the complex manifold

$$
N=\left\{\left(z_{1}, z_{2}, z_{3}\right) \in \mathbb{C}^{3} \mid z_{1}^{2}+z_{2}^{2}+z_{3}^{2}=1\right\}
$$

and the holomorphic 2-form on $\mathbb{C}^{3}$ :

$$
\eta=\frac{1}{4 \pi^{2}}\left(z_{1} \mathrm{~d} z_{2} \wedge \mathrm{d} z_{3}+z_{2} \mathrm{~d} z_{3} \wedge \mathrm{d} z_{1}+z_{3} \mathrm{~d} z_{1} \wedge \mathrm{d} z_{2}\right) .
$$

It is clear that the pull back of $\eta$ defines a holomorphic closed 2-form on $N$. By $\eta_{1}$ and $\eta_{2}$, we denote the real part and the imaginary part of $\eta$, respectively. Consider the submanifold 
$\mathbb{R}^{3}$ of $\mathbb{C}^{3}$ defined by $\mathbb{R}^{3} \cong\left\{\left(z_{1}, z_{2}, z_{3}\right) \mid y_{1}=y_{2}=y_{3}=0\right\}$. The intersection $S$ of $N$ with $\mathbb{R}^{3}$ is a unit sphere:

$$
S=N \cap \mathbb{R}^{3}=\left\{\left(x_{1}, x_{2}, x_{3}\right) \in \mathbb{R}^{3} \mid x_{1}^{2}+x_{2}^{2}+x_{3}^{2}=1\right\}
$$

The pull back of $\eta_{1}$ to $S$ is a real valued 2-form, which takes the same expression:

$$
\left.\eta_{1}\right|_{S}=\frac{1}{4 \pi^{2}}\left(x_{1} \mathrm{~d} x_{2} \wedge \mathrm{d} x_{3}+x_{2} \mathrm{~d} x_{3} \wedge \mathrm{d} x_{1}+x_{3} \mathrm{~d} x_{1} \wedge \mathrm{d} x_{2}\right) .
$$

It is clear that $\eta_{1}$ is a volume form on the unit sphere: $\int_{S} \eta_{1}=1$. On the other hand, the pull back of $\eta_{2}$ to $S$ vanishes.

Let $X=N \times N$, and $\omega=\sqrt{2} p_{1}^{*} \eta+p_{2}^{*} \eta$, where $p_{1}, p_{2}$ stand for the projections on the first and second components. Then $\omega$ is a holomorphic closed 2-form on $X$. It is simple to see that the group of periods of $\left(\omega_{1}, \omega_{2}\right)$ contains $(1,0)$ and $(\sqrt{2}, 0)$, and therefore is not discrete. Hence, the corresponding holomorphic Lie algebroid $A$ is not integrable.

We end this section with the following

Proposition 3.20. Let $\Gamma \rightrightarrows X$ and $\Gamma^{\prime} \rightrightarrows X^{\prime}$ be holomorphic Lie groupoids with holomorphic Lie algebroids $A \rightarrow X$ and $A^{\prime} \rightarrow X^{\prime}$, respectively. Assume that $\phi: \Gamma \rightarrow \Gamma^{\prime}$ is a homomorphism of the underlying real Lie groupoids and its infinitesimal Lie $(\phi): A \rightarrow A^{\prime}$ is a morphism of holomorphic Lie algebroids. Then $\phi$ is a homomorphism of holomorphic Lie groupoids.

Proof. The map Lie $(\phi): A \rightarrow A^{\prime}$ is holomorphic implies that the following

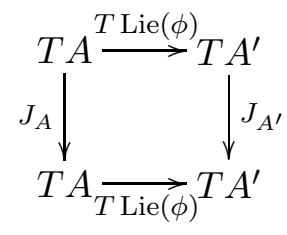

is a commutative diagram of Lie algebroid morphisms.

Hence it implies the commutativity of the Lie groupoid homomorphisms of the diagram below:

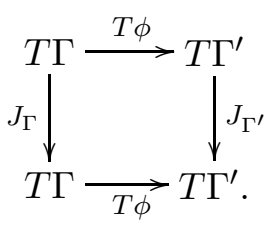

The latter exactly means that $\phi: \Gamma \rightarrow \Gamma^{\prime}$ is a holomorphic map.

\subsection{Holomorphic symplectic groupoids}

As an application, in this section, we study holomorphic symplectic groupoids.

Holomorphic symplectic groupoids can be introduced in a similar fashion as in the smooth case. 
Definition 3.21. A holomorphic symplectic groupoid is a holomorphic Lie groupoid $\Gamma \rightrightarrows X$ together with a holomorphic symplectic 2-form $\omega \in \Omega^{2,0}(\Gamma)$ such that the graph of multiplication $\Lambda \subset \Gamma \times \Gamma \times \bar{\Gamma}$ is a Lagrangian submanifold, where $\bar{\Gamma}$ stands for the $\Gamma$ equipped with the opposite symplectic structure.

As in the smooth case, this last condition is equivalent to $\partial \omega=0$ where $\partial: \Omega^{2}(\Gamma) \rightarrow \Omega^{2}\left(\Gamma_{2}\right)$ is the alternate sum of the pull back maps of the three face maps $\Gamma_{2} \rightarrow \Gamma$. In this case, $\omega$ is said to be multiplicative.

As in the smooth case, if $\Gamma \rightrightarrows X$ is a holomorphic symplectic groupoid, then $X$ is naturally a holomorphic Poisson manifold. More precisely, there exists a unique holomorphic Poisson structure on $X$ such that the source map $s: \Gamma \rightarrow X$ is a holomorphic Poisson map, while the target map is then an anti-Poisson map.

Given a holomorphic symplectic groupoid $\Gamma \rightrightarrows X$, its holomorphic Lie algebroid is isomorphic to the cotangent Lie algebroid $\left(T^{*} X\right)_{\pi} \rightarrow X$, where $\pi$ is the induced holomorphic Poisson structure on $X$.

Conversely, a holomorphic Poisson manifold $(X, \pi)$ is said to be integrable if it is the induced holomorphic Poisson structure on the unit space of a holomorphic symplectic groupoid $\Gamma \rightrightarrows X$. We say that $\Gamma \rightrightarrows X$ integrates the holomorphic Poisson structure $(X, \pi)$.

The main theorem is the following:

Theorem 3.22. A holomorphic Poisson manifold is integrable if, and only if, either its real or its imaginary part is integrable as a real Poisson manifold.

More precisely, if $\left(\Gamma \rightrightarrows X, \omega_{R}+i \omega_{I}\right)$ is a holomorphic symplectic groupoid integrating the holomorphic Poisson structure $\left(X, \pi_{R}+i \pi_{I}\right)$, then $\left(\Gamma \rightrightarrows X, 4 \omega_{R}\right)$ and $\left(\Gamma \rightrightarrows X,-4 \omega_{I}\right)$ are symplectic groupoids integrating the real Poisson manifolds $\left(X, \pi_{R}\right)$ and $\left(X, \pi_{I}\right)$, respectively.

Conversely given a holomorphic Poisson manifold $(X, \pi)$, where $\pi=\pi_{R}+i \pi_{I} \in \Gamma\left(\wedge^{2} T^{1,0} X\right)$, if $\left(\Gamma \rightrightarrows X, \omega_{R}\right)$ is a s-connected and s-simply connected symplectic groupoid integrating $\left(X, \pi_{R}\right)$, then

(a) $\Gamma \rightrightarrows X$ admits a holomorphic Lie groupoid structure. By $J_{\Gamma}: T \Gamma \rightarrow T \Gamma$ we denote its almost complex structure;

(b) $\quad\left(\Gamma \rightrightarrows M, \omega_{I}\right)$, where $\omega_{I}(\cdot, \cdot):=\omega_{R}\left(J_{\Gamma} \cdot, \cdot\right)$, is a symplectic groupoid integrating $\left(X, \pi_{I}\right)$;

(c) $\quad(\Gamma \rightrightarrows X, \omega)$, where $\omega:=\frac{1}{4}\left(\omega_{R}-i \omega_{I}\right)$, is a holomorphic symplectic groupoid integrating $(X, \pi)$.

This theorem can be derived from Theorem 5.2 in [20] or Theorem 3.4 in [5] via the equivalence relation between holomorphic Poisson manifolds and Poisson Nijenhuis structures established in Theorem 2.5. We, however, give a direct proof below, as an application of Theorem 3.22, We start with a simple fact of complex geometry.

Lemma 3.23. Let $X$ be a complex manifold with almost complex structure $J$ and $\alpha_{R} \in \Omega^{2}(X)$ a two-form on the underlying real manifold. By $\alpha_{R}^{b}$ we denote its induced bundle map $\alpha_{R}^{b}$ : $T X \rightarrow T^{*} X$. 
(a) If $J^{*} \circ \alpha_{R}^{b}=\alpha_{R}^{b} \circ J$, then $\alpha(\cdot, \cdot):=\alpha(\cdot, \cdot)-i \alpha(J \cdot, \cdot)$ is a $(2,0)$-form on $X$.

(b) If, moreover, $\alpha_{R}^{b}: T X \rightarrow T^{*} X$ is a holomorphic map, then $\alpha$ is a holomorphic 2-form.

(c) Furthermore, $d \alpha_{R}=0$ iff $\partial \alpha=0$.

Proof of Theorem 3.22. Assume that $\left(\Gamma \rightrightarrows X, \omega_{R}+i \omega_{I}\right)$ is a holomorphic symplectic groupoid integrating the holomorphic Poisson structure $\left(X, \pi_{R}+i \pi_{I}\right)$. Clearly, both $\left(\Gamma \rightrightarrows X, \omega_{R}\right)$ and $\left(\Gamma \rightrightarrows X, \omega_{I}\right)$ are symplectic groupoids. $\mathrm{By} \Pi_{R}$ and $\Pi_{I}$ we denote the Poisson tensors corresponding to the symplectic two-forms $\omega_{R}$ and $\omega_{I}$, respectively. Then the holomorphic Poisson bivector field corresponding to $\omega$ is $\frac{1}{4}\left(\Pi_{R}-i \Pi_{I}\right)$. A Poisson map between two holomorphic Poisson manifolds is also a Poisson map between their real parts, and as well as between their imaginary parts. As a consequence, we have $\frac{1}{4} s_{*} \Pi_{R}=\pi_{R}$ and $-\frac{1}{4} s_{*} \Pi_{I}=\pi_{I}$. It follows that $\left(\Gamma \rightrightarrows X, 4 \omega_{R}\right)$ and $\left(\Gamma \rightrightarrows X,-4 \omega_{I}\right)$ are symplectic groupoids integrating, respectively, the real part $\pi_{R}$ and the imaginary part $\pi_{I}$ of $\pi$.

Conversely, let $(X, \pi)$, where $\pi=\pi_{R}+i \pi_{I} \in \Gamma\left(\wedge^{2} T^{1,0} X\right)$, be a holomorphic Poisson manifold and $\left(\Gamma \rightrightarrows X, \omega_{R}\right)$ a $s$-connected and $s$-simply connected symplectic groupoid integrating $\left(X, \pi_{R}\right)$. Hence the Lie algebroid of $\Gamma \rightrightarrows X$ is isomorphic to $\left(T^{*} X\right)_{\pi_{R}}$. By Proposition 2.6, $\left(T^{*} X\right)_{\pi_{R}}$ is the underlying real Lie algebroid of the holomorphic Lie algebroid $\left(T^{*} X\right)_{\frac{1}{4} \pi}$. According to Theorem 3.17 $\Gamma \rightrightarrows X$ admits a multiplicative almost complex structure $J_{\Gamma}$ which makes $\Gamma$ into a holomorphic Lie groupoid.

For the remaining of the proof, we need recall some well known facts concerning Poisson groupoids [16, 17]. For a Poisson groupoid $(\Gamma \rightrightarrows X, \Pi)$, the map

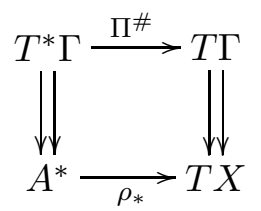

induced by the Poisson tensor $\Pi$ is a Lie groupoid homomorphism, where $\left(A, A^{*}\right)$ denotes its corresponding Lie bialgebroid with anchors $\rho$ and $\rho_{*}$, respectively. Here $T \Gamma \rightrightarrows T X$ and $T^{*} \Gamma \rightrightarrows A^{*}$ are, respectively, the tangent and cotangent Lie groupoids of $\Gamma \rightrightarrows X[3]$. The infinitesimal Lie(П) of Eq. (21) is the Lie algebroid homomorphism below (Theorem 8.3 in [16]):

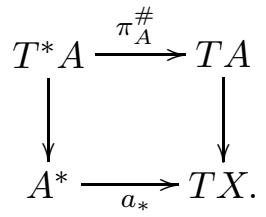

where the left hand side $T^{*} A \longrightarrow A^{*}$ is the cotangent Lie algebroid $T^{*}\left(A^{*}\right) \longrightarrow A^{*}$ induced by the Lie Poisson structure on $A^{*}$ by identifying $T^{*} A$ with $T^{*}\left(A^{*}\right)$ via the canonical isomorphism $R: T^{*} A^{*} \longrightarrow T^{*} A$ as given in Theorem 5.5 of [16], the right hand side $T A \longrightarrow T X$ is the tangent Lie algebroid of $A$, and $\pi_{A}$ is the Lie Poisson tensor on $A$.

Now consider the symplectic groupoid $\left(\Gamma \rightrightarrows X, \omega_{R}\right)$. Its corresponding Lie bialgebroid is $\left(\left(T^{*} X\right)_{\pi_{R}}, T X\right)$, and the Lie Poisson structure on $T^{*} X$ dual to the Lie algebroid $T X$ is the 
canonical symplectic structure on the cotangent bundle. Let $\alpha \in \Omega^{2}\left(T^{*} X\right)$ be its symplectic form. Applying the above general result on Poisson groupoids and reversing the maps, we have a Lie groupoid homomorphism

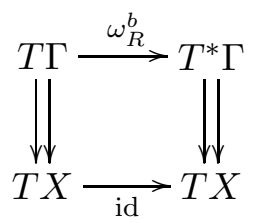

Its infinitesimal $\operatorname{Lie}\left(\omega_{R}^{b}\right)$ is the Lie algebroid homomorphism:

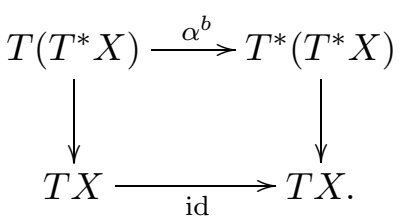

So far we have ignored the complex structures, and all the maps involved in Eqs. (22) and (23) are considered as maps of real manifolds. On the other hand, $T \Gamma \rightrightarrows T X$ and $T^{*} \Gamma \rightrightarrows$ $T X$ are both holomorphic Lie groupoids since $\Gamma \rightrightarrows X$ is a holomorphic Lie groupoid. And their corresponding holomorphic Lie algebroids $T^{*}\left(T^{*} X\right) \rightarrow T X$ and $T\left(T^{*} X\right) \rightarrow T X$ are, respectively, the holomorphic counterparts of the Lie algebroids involved in Eq. (23). It is easy to see that $\alpha^{b}$ is a holomorphic map, and therefore is indeed a holomorphic Lie algebroid homomorphism. By Proposition 3.20, it follows that $\omega_{R}^{b}$ is indeed a homomorphism of holomorphic Lie groupoids. Moreover, the diagram

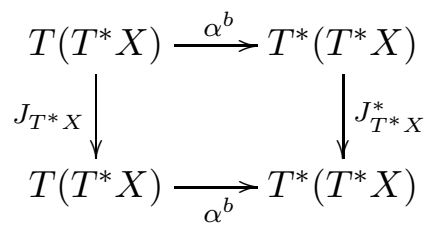

is a commutative diagram of real Lie algebroid homomorphisms, since $\alpha$ is essentially the real part of the canonical holomorphic symplectic 2 -form on $T^{*} X$. Hence we have a commutative diagram of Lie groupoid homomorphisms:

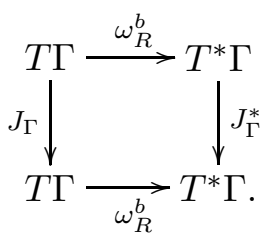

Therefore, by Lemma 3.23, we conclude that $\omega=\omega_{R}-i \omega_{I} \in \Omega^{2,0}(\Gamma)$, where $\omega_{I}(\cdot, \cdot)=\omega(J \cdot, \cdot)$, is a holomorphic closed 2 -form. It is clear that $\omega$ is non-degenerate and multiplicative. Hence ( $\Gamma \rightrightarrows X, \omega)$ is a holomorphic symplectic groupoid. Finally we have

$$
s_{*}\left(\omega_{R}^{b} J_{\Gamma}\right)^{-1} s^{*}=-s_{*} J_{\Gamma}\left(\omega_{R}^{b}\right)^{-1} s^{*}=-J_{X} s_{*}\left(\omega_{R}^{b}\right)^{-1} s^{*}=-J_{X} \pi_{R}^{\#}=\pi_{I}^{\#} .
$$

The conclusion thus follows. 
Indeed the exact same proof as above leads to the following

Theorem 3.24. Let $(X, \pi)$, where $\pi=\pi_{R}+i \pi_{I} \in \Gamma\left(\wedge^{2} T^{1,0} X\right)$, be a holomorphic Poisson manifold. Assume that $\Gamma \rightrightarrows X$ is a holomorphic Lie groupoid with almost complex structure $J_{\Gamma}$, whose corresponding holomorphic Lie algebroid is $\left(T^{*} X\right)_{\frac{1}{4} \pi}$. Moreover, assume that there exists a symplectic real 2-form $\omega_{R}$ on the underlying real Lie groupoid such that $\left(\Gamma \rightrightarrows X, \omega_{R}\right)$ is a symplectic groupoid integrating the real Poisson structure $\pi_{R}$. Then $\omega=\omega_{R}-i \omega_{I} \in$ $\Omega^{2,0}(\Gamma)$, where $\omega_{I}(\cdot, \cdot)=\omega(J \cdot, \cdot)$, is a multiplicative holomorphic symplectic 2-form on $\Gamma$ so that $\left(\Gamma \rightrightarrows X, \frac{1}{4} \omega\right)$ is a holomorphic symplectic groupoid integrating the holomorphic Poisson structure $\pi$.

\subsection{Holomorphic extension of analytic Poisson structures}

To any analytic Poisson structure $\pi_{\text {real }}$ on $\mathbb{R}^{n}$, there associates a canonical holomorphic Poisson structure $\pi$ on $\mathbb{C}^{n}$, called its holomorphic extension, which can be defined as follows. Assume that the Poisson brackets of $\pi_{\text {real }}$, on the canonical coordinates, are given by

$$
\left\{x_{i}, x_{j}\right\}=\phi_{i j}\left(x_{1}, \ldots, x_{n}\right),
$$

where $\phi_{i j}$ are, for all $i, j=1, \ldots, n$, real analytic functions on $\mathbb{R}^{n}$. Then the Poisson brackets of its holomorphic extension on $\mathbb{C}^{n}$ are given by

$$
\left\{z_{i}, z_{j}\right\}=\phi_{i j}\left(z_{1}, \cdots, z_{n}\right) .
$$

Then we have the following:

Theorem 3.25. If the holomorphic extension $\pi$ of a real analytic Poisson structure $\pi_{\text {real }}$ is integrable, then $\pi_{\text {real }}$ must be integrable.

Let $\pi_{R}$ and $\pi_{I}$ be the real part and the imaginary part of $\pi$. We advise the reader to keep in mind that $\pi_{R}$ is a smooth bivector field on $\mathbb{C}^{n}$, and not to confuse it with $\pi_{\text {real }}$, which is a smooth bivector field on $\mathbb{R}^{n}$.

Recall that a Poisson involution on a Poisson manifold $P$ is a Poisson diffeomorphism $\Phi$ : $P \longrightarrow P$ such that $\Phi^{2}=i d$.

Lemma 3.26. The complex conjugation map $\sigma\left(z_{1}, \cdots, z_{n}\right)=\left(\overline{z_{1}}, \cdots, \overline{z_{n}}\right)$ is a Poisson involution of $\left(\mathbb{C}^{n}, \pi_{R}\right)$.

Proof. We write $\phi_{i j}=f_{i j}+i g_{i j}$, where $f_{i j}, g_{i j} \in C^{\infty}\left(\mathbb{C}^{n}, \mathbb{R}\right)$, and $z_{k}=x_{k}+i y_{k}, k=1, \cdots, n$. One computes immediately that

$$
\begin{aligned}
\pi_{R}= & \frac{1}{4} \sum_{i, j=1}^{n} f_{i j}\left(z_{1}, \ldots, z_{n}\right)\left(\frac{\partial}{\partial x_{i}} \wedge \frac{\partial}{\partial x_{j}}-\frac{\partial}{\partial y_{i}} \wedge \frac{\partial}{\partial y_{j}}\right) \\
& +\frac{1}{4} \sum_{i, j=1}^{n} g_{i j}\left(z_{1}, \ldots, z_{n}\right)\left(\frac{\partial}{\partial x_{i}} \wedge \frac{\partial}{\partial y_{j}}+\frac{\partial}{\partial y_{i}} \wedge \frac{\partial}{\partial x_{j}}\right) .
\end{aligned}
$$

By constructions, we have $\sigma^{*} \phi_{i j}=\overline{\phi_{i j}}$. Thus it follows that $\forall i, j=1, \cdots, n$,

$$
\sigma^{*} f_{i j}=f_{i j}, \quad \sigma^{*} g_{i j}=-g_{i j} .
$$


On the other hand, it is obvious that

$$
\sigma_{*}\left(\frac{\partial}{\partial x_{j}}\right)=\frac{\partial}{\partial x_{j}}, \quad \sigma_{*}\left(\frac{\partial}{\partial y_{j}}\right)=-\frac{\partial}{\partial y_{j}}
$$

The conclusion thus follows immediately.

Remark 3.27. Note that $\sigma$ is an anti-Poisson map with respect to the imaginary part $\pi_{I}$ of $\pi$.

It is well known that the stable locus of a Poisson involution carries a natural Poisson structure [11, 22. More precisely, let $Q$ be the stable locus of a Poisson involution $\Phi: P \longrightarrow P$. Assume that the Poisson tensor $\pi$ on $P$ is $\pi=\sum_{i} X_{i} \wedge Y_{i}$, where $X_{i}$ and $Y_{i}$ are vector fields on $P$. Then the induced Poisson tensor $\pi_{Q}$ on $Q$ is given by

$$
\pi_{Q}=\left.\sum_{i} X_{i}^{+} \wedge Y_{i}^{+}\right|_{Q}
$$

where $X_{i}^{+}$and $Y_{i}^{+}$are vector fields on $P$ defined by $X_{i}^{+}=\frac{1}{2}\left(X_{i}+\Phi_{*} X_{i}\right)$ and $Y_{i}^{+}=\frac{1}{2}\left(Y_{i}+\right.$ $\left.\Phi_{*} Y_{i}\right)$, respectively. The Poisson manifold $\left(Q, \pi_{Q}\right)$ is called a Dirac submanifold of $(P, \pi)$ (which is also called a Lie-Dirac submanifold in [7]).

The following result was proved in [22] (see also [10, 7]).

Proposition 3.28. If $Q$ is the stable locus of a Poisson involution on an integrable Poisson manifold $P$, then $Q$ is always an integrable Poisson manifold itself.

Lemma 3.29. Let $\pi$ be the holomorphic extension on $\mathbb{C}^{n}$ of an analytic Poisson structure $\pi_{\text {real }}$ on $\mathbb{R}^{n}$. The induced Poisson structure on the stable locus of the Poisson involution $\sigma$ on $\left(\mathbb{C}^{n}, \pi_{R}\right)$ is isomorphic to $\left(\mathbb{R}^{n}, \frac{1}{4} \pi_{\text {real }}\right)$.

Proof. Let $Q$ be the stable locus of $\sigma$. Then $Q=\left\{\left(z_{1}, \cdots, z_{n}\right) \mid y_{1}=\cdots=y_{n}=0\right\} \cong \mathbb{R}^{n}$. By Eqs. (24) and (26), we have

$$
\left(\pi_{R}\right)_{Q}=\left.\frac{1}{4} \sum_{i, j=1}^{n} f_{i j}\left(z_{1}, \ldots, z_{n}\right)\right|_{Q} \frac{\partial}{\partial x_{i}} \wedge \frac{\partial}{\partial x_{j}} .
$$

Since $\left.f_{i j}\left(z_{1}, \cdots, z_{n}\right)\right|_{Q}=\phi_{i j}\left(x_{1}, \cdots, x_{n}\right)$, the right hand side is equal to $\frac{1}{4} \pi_{\text {real }}$.

Proof of Theorem 3.25, It follows immediately from Theorem 3.22, Proposition 3.28 and Lemma 3.29.

Example 3.30. Consider the holomorphic Poisson structure

$$
\pi=e^{\frac{z_{1}^{2}+z_{2}^{2}+z_{3}^{2}}{2}} z_{1} \frac{\partial}{\partial z_{2}} \wedge \frac{\partial}{\partial z_{3}}+\text { c.p. }
$$

on $\mathbb{C}^{3}$. It is clear that this is a holomorphic extension of the analytic Poisson structure:

$$
\pi_{\text {real }}=e^{\frac{x_{1}^{2}+x_{2}^{2}+x_{3}^{2}}{2}} x_{1} \frac{\partial}{\partial x_{2}} \wedge \frac{\partial}{\partial x_{3}}+c . p .
$$

on $\mathbb{R}^{3}$.

According to Example 3.3 in [8], the Poisson structure (28) is not integrable. By Theorem 3.25, the holomorphic Poisson structure (27) on $\mathbb{C}^{3}$ is not integrable either. 


\section{References}

[1] M. N. Boyom, KV-cohomology of Koszul-Vinberg algebroids and Poisson manifolds, Internat. J. Math.16 (2005), no. 9, 1033-1061.

[2] S. Canez, Private communication.

[3] A. Coste, P. Dazord, and A. Weinstein, Groupoïdes symplectiques, Publications du Département de Mathématiques. Nouvelle Série. A, Vol. 2, Publ. Dép. Math. Nouvelle Sér. A, 87, Univ. Claude-Bernard, Lyon, 1987, pp. i-ii, 1-62.

[4] A. Cattaneo and G. Felder, Poisson sigma models and symplectic groupoids, Prog. Math. 198 (2001) 61-93,

[5] M. Crainic, Generalized complex structures and Lie brackets arXiv:math/0412097

[6] M. Crainic and R.-L. Fernandes, Integrability of Lie brackets. Ann. of Math. (2) 157 (2003), 575-620.

[7] M. Crainic and R.-L. Fernandes, Integrability of Poisson brackets, J. Differential Geometry. 66 (2004), 71-137.

[8] M. Crainic and R.L. Fernandes, Lectures on integrability of Lie brackets, arXiv:0611259v1

[9] S. Evens, J.-H. Lu, and A. Weinstein, Transverse measures, the modular class, and a cohomology pairing for Lie algebroids, Quart. J. Math. Oxford (2) 50 (1999), 417-436. arXiv:dg-ga/9610008v1

[10] R.L. Fernandes, A note on proper Poisson actions, arXiv:0503147.

[11] Fernandes, R., and Vanhaecke, P., Hyperelliptic Prym varieties and integrable systems, Commun. Math. Phys.221 (2001) 169-196.

[12] J. Huebschmann, Duality for Lie-Rinehart algebras and the modular class, J. Reine Angew. Math.510 (1999), 103-159.

[13] Y. Kosmann-Schwarzbach, The Lie bialgebroid of a Poisson-Nijenhuis manifold. Lett. Math. Phys., 38(4):421-428, 1996.

[14] Y. Kosmann-Schwarzbach and F. Magri, Poisson-Nijenhuis structures. Ann. Inst. H. Poincaré Phys. Théor., 53(1):35-81, 1990.

[15] C.-G. Laurent, M. Stienon, and P. Xu, Holomorphic Poisson manifolds and holomorphic Lie algebroids, Preprint 2008.

[16] K. C. H. Mackenzie and P. Xu, Lie bialgebroids and Poisson groupoids. Duke Math. J., 73(2):415-452, 1994.

[17] K. C. H. Mackenzie and P. Xu, Integration of Lie bialgebroids. Topology, 39(3):445-467, 2000 . 
[18] F. Magri and C. Morosi, On the reduction theory of the Nijenhuis operators and its applications to Gelfand-Dikil equations, Proceedings of the IUTAM-ISIMM symposium on modern developments in analytical mechanics, Vol. II (Torino, 1982), 117, 1983, pp. 599-626.

[19] F. Magri and C. Morosi, Old and new results on recursion operators: an algebraic approach to KP equation, Topics in soliton theory and exactly solvable nonlinear equations (Oberwolfach, 1986), World Sci. Publishing, Singapore, 1987, pp. 78-96.

[20] M. Stiénon and P. Xu, Poisson quasi-Nijenhuis manifolds. Comm. Math. Phys. 27 (2007), 709-725.

[21] A. Weinstein, The integration problem for complex Lie algebroids, From Geometry to Quantum Mechanics, in Honor of Hideki Omori, Y. Maeda, T. Ochiai, P. Michor, and A. Yoshioka, eds., Progress in Mathematics, Birkhäuser, New York (2007), 93-109.

[22] P. Xu, Dirac submanifolds and Poisson involutions, Ann. Scient. Ec. Norm. Sup. 36 (2003), 403-430. 